Proyecciones Journal of Mathematics

Vol. 34, No 1, pp. 1-13, March 2015.

Universidad Católica del Norte

Antofagasta - Chile

\title{
Complementary nil vertex edge dominating sets
}

\author{
S. V. Siva Rama Raju \\ Ibra College of Technology, Sultanate of Oman \\ and \\ I. H. Nagaraja Rao \\ G. V. P. College for P. G. Courses, India \\ Received: July 2012. Accepted : September 2014
}

\begin{abstract}
Dominating sets play a vital role in day-to-day life problems. For providing effective services in a location, central points are to be identified. This can easily be achieved by graph theoretic techniques. Such graphs and relevant parameters are introduced and extensively studied. One such parameter is complementary nil vertex edge dominating set(cnved-set). A vertex edge dominating set(ved-set) of a connected graph $G$ with vertex set $V$ is said to be a complementary nil vertex edge dominating set(cnved-Set) of $G$ if and only if $V-D$ is not a ved-set of $G$. A cnved-set of minimum cardinality is called a minimum cnvedset(mcnved-set) of $G$ and this minimum cardinality is called the complementary nil vertex-edge domination number of $G$ and is denoted by $\gamma_{\text {cnve }}(G)$. We have given a characterization result for a ved-set to be a cnved-set and also bounds for this parameter are obtained.
\end{abstract}

Subject Classification: 05C69.

Keywords: Complementary nil vertex edge domination, complementary nil vertex edge domination number, connected domination. 


\section{Introduction \& Preliminaries}

Domination is an active topic in graph theory and has numerous applications to distributed computing, the web graph and adhoc networks. Haynes et al.[3] gave a comprehensive introduction to the theoretical and applied facets of domination in graphs.

A subset $S$ of the vertex set $V$ of $G$ is said to be a dominating set of $G$ if each vertex in $V-D$ is adjacent to some vertex of $D$. The domination number $\gamma(G)$ is the minimum cardinality of the dominating set of $G[3]$. A subset $E^{\prime}$ of the edge set $E$ of a graph $G$ is said to be an edge dominating set of $G$ if each edge in $E-E^{\prime}$ is adjacent to some edge in $E^{\prime}$. The edge domination number $\gamma^{\prime}(G)$ is the minimum cardinality of the edge dominating set of $G[3]$. A subset $D$ of vertices is said to be a vertex edge dominating set of $G$ if each edge in $G$ has either one of its ends from $D$ (or) one of its ends is adjacent to a vertex in $D$. The vertex edge domination number $\gamma_{v e}(G)$ is the minimum cardinality of the vertex edge dominating set of $G[5]$. A subset $D$ of vertices is said to be a complementary nil dominating set of $G$ if $V-D$ is not a dominating set of $G$. The minimum cardinality of a complementary nil dominating set is called a complementary nil domination number of $G$ and is denoted by $\gamma_{c n d}(G)[6]$.

Many variants of vertex - vertex dominating sets have been studied. In the present paper, we introduce a new variant of vertex-edge dominating set namely complementary nil vertex edge dominating set. We have given the characterization result for vertex edge dominating set to be complementary nil vertex edge dominating set and characterized the graphs of order $n$ having cnved number $n$, characterized trees of order $n$ having cnved numbers $n-1, n-2$. Bounds for this parameter are also obtained.

All graphs considered in this paper are simple, finite, undirected and connected. For standard terminology and notation, we refer Bondy \& Murthy[1].

\section{Characterization and other relevant results}

In this section, we mainly obtain characterization result for a proper subset of the vertex set of $G$ to be a cnved-set of $G$.

Theorem 2.1. A ved - set of a (connected)graph $G$ is a cnved-set iff there is an edge $e_{1} \in F=\{e=u v \in E(G): u, v \in D\}$ with $N\left[e_{1}\right] \subseteq F$.

Proof: Let $D$ be a cnved-set of $G$. So $V-D$ is not a ved-set of $G$. So there is an edge, say $e_{1}$ with ends $u_{1}, v_{1}$ of $G$ such that $u_{1}, v_{1} \notin V-D$ 
and $N\left(u_{1}\right) \bigcap(V-D)=\phi=N\left(v_{1}\right) \bigcap(V-D) . \Rightarrow u_{1}, v_{1} \in D, N\left(u_{1}\right) \subseteq$ $D \& N\left(v_{1}\right) \subseteq D \Rightarrow N\left[e_{1}\right] \subseteq F$. Conversely assume that there is an $e=$ $u_{1} v_{1} \in F$ such that $N[e] \subseteq F \Rightarrow u_{1}, v_{1} \in D \& N\left(u_{1}\right), N\left(v_{1}\right) \subseteq D$. So there is no $w_{1}$ in $V-D$ such that atleast one of $u_{1}, v_{1}$ is adjacent with $w_{1}$ in $G$. Hence $V-D$ is not a ved-set of $G$. Thus $D$ is a cnved-set of $G$.

Note: For a cnved-set there is an edge of $G$ whose ends are in cnved-set. So cnved- set is not an independent set in $G$.

Corollary 2.2. Let $D$ be a cnved-set of a (connected)graph $G$. Then $D$ has atleast two enclaves in $D$.

Proof: By the above Characterization Result, there is atleast one edge , say $e_{1}=u_{1} v_{1}$ in $F=\{e=u v \in E(G): u, v \in D\}$ with $N\left(e_{1}\right) \subseteq F \Rightarrow$ $N\left(u_{1}\right) \subseteq D \& N\left(v_{1}\right) \subseteq D$. Hence $u_{1} \& v_{1}$ are enclaves in $D$.

Result 2.3. If $D_{1}, D_{2}$ are ved - sets such that atleast one of them is a cnved-set then $D_{1} \cap D_{2} \neq \phi$.

Proof: If $D_{1} \cap D_{2}=\phi$ then $D_{1} \subseteq V-D_{2}$ and $D_{2} \subseteq V-D_{1} \Rightarrow$ Both $D_{1}, D_{2}$ are not cnved-sets and this contradicts the hypothesis.

We now give the bounds for cnved number of connected graphs.

Theorem 2.4 If $G$ is a connected graph, then

$$
\gamma_{v e}(G)+2 \leq \gamma_{c n v e}(G)
$$

Proof: Let $D$ be a minimum cnved-set of $G$. By the Characterization Result there is an edge $e_{1}=u_{1} v_{1}$ in $F$ such that $N\left[e_{1}\right] \subseteq F$. Hence follows that $D-\left\{u_{1}, v_{1}\right\}$ is a ved-set of $G$. Thus $\gamma_{v e}(G) \leq|D|-2=\gamma_{c n v e}(G)-2$, which implies $\gamma_{v e}(G)+2 \leq \gamma_{\text {cnve }}(G)$.

Furthermore the lower bound is attained in the case of $P_{8}$. Hence the bound is sharp.

A lower bound for $\gamma_{\text {cnve }}(G)$ is obtained in terms of the number of edges $(\epsilon)$ and maximum degree $\Delta(G)$ of the vertices of $G$.

Corollary 2.5. For any graph $G\left(\neq K_{2}\right),\left\lceil\frac{\epsilon}{\Delta(G)(\Delta(G)-1)}\right\rceil+2 \leq \gamma_{\text {cnve }}(G)$ ( $\epsilon$ being the number of edges of $G$ ). 
Proof: Clearly $\Delta(G) \geq 2$. Any vertex $v$ can have atmost $\Delta(G)$ neighbours. Furthermore any neighbour $u$ of $v$ can dominate atmost $\Delta(G)-1$ edges(excluding the edge $u v$ )

$$
\Rightarrow\left\lceil\frac{\epsilon}{\Delta(G)(\Delta(G)-1)}\right\rceil \leq \gamma_{v e}(G) .
$$

Then by the above Theorem the inequality follows.

Note: The bound is sharp as it is attained in the case of $C_{4}$. For any $k$ regular graph $G\left(\neq K_{2}\right)$ with $n$ vertices ,

$$
\left\lceil\frac{n}{k-1}\right\rceil+2 \leq \gamma_{\text {cnve }}(G)
$$

Proof: The proof follows from the above theorem and the fact that

$$
k \geq 2, \Delta(G)=k, \epsilon=n k .
$$

Theorem 2.7. If $G$ is a connected graph of order $n$ and having $\epsilon$ edges, then

$$
\left\lceil\frac{2 \epsilon-n^{2}+5 n}{4}\right\rceil \leq \gamma_{\text {cnve }}(G)
$$

Proof: Let $S$ be a minimum cnved-set of $G$. Since $V-S$ is not a cnvedset of $G$, there is an edge $u v$ such that $N[u v] \subseteq S$. Each of $u, v$ are non adjacent to all the vertices in $V-S$. So

$$
\begin{array}{r}
\epsilon \leq n_{C_{2}}-2\left(n-\gamma_{\text {cnve }}(G)\right) \\
\Rightarrow\left\lceil\frac{2 \epsilon-n^{2}+5 n}{4}\right\rceil \leq \gamma_{\text {cnve }}(G)
\end{array}
$$

Note: The bound is sharp as it is attained in the case of $\left\langle v_{4} v_{1} v_{2} v_{3} v_{1}\right\rangle$.

Theorem 2.8 For a connected graph $G$ with $g(G) \neq 3,2 \delta(G) \leq \gamma_{\text {cnve }}(G)$ 
Proof: Let $S$ be a minimum cnved-set of $G$. Since $V-S$ is not a cnved-set of $G$, there is an edge $e=u v$ such that $N[e] \subseteq S$. That is $|N[e]| \leq|S|$. Hence $2 \delta(G) \leq \gamma_{\text {cnve }}(G)$.

Note: The bound is sharp as it is attained in the case of $C_{5}$.

Corollary 2.9 For a connected Bipartite graph $G, 2 \delta(G) \leq \gamma_{\text {cnve }}(G)$.

Proof: The proof follows from the fact that $G$ cannot have odd cycles.

Note The bound is sharp as it is attained in the case of $C_{4}$.

Theorem 2.10. If $G$ is a connected graph, then

$$
\gamma_{c n v e}(G) \leq \gamma_{v e}(G)+2 \Delta(G)
$$

Proof: Let $D$ be the minimum vertex edge dominating set for $G$. Then for any edge $e$ in $G, D \cup N[e]$ is a cnved-set of $G$. Hence

$$
\begin{aligned}
& \quad \gamma_{\text {cnve }}(G) \leq|D \bigcup N[e]| \\
& \leq|D|+d(u)+d(v) \\
& \leq \gamma_{v e}(G)+2 \Delta(G)
\end{aligned}
$$

Note: The bound is sharp as it is attained in the case of $C_{6}$.

Remark: Since $\gamma_{v e}(G) \leq \gamma(G)$, follows that $\gamma_{c n v e}(G) \leq \gamma(G)+2 \Delta(G)$

Theorem 2.11. If $G$ is a connected graph having a pendant vertex, then $\gamma_{\text {cnve }}(G) \leq \gamma_{v e}(G)+\Delta(G)$.

Proof: Suppose that $D$ is a minimum vertex edge dominating set for $G$. Let $v$ be adjacent to pendant vertex $u$ (say) in $G$. Clearly $D \bigcup N[v]$ is a cnved-set for $G$. Hence

$$
\gamma_{\text {cnve }}(G) \leq|D \bigcup N[v]|
$$


$\leq \gamma_{v e}(G)+\Delta(G)$

Note: The bound is sharp as it is attained in the case of $P_{5}$.

Corollary 2.12. If $G=P_{n}$, then $\gamma_{c n v e}(G) \leq \gamma_{v e}(G)+2$.

Proof: Since $\Delta(G)=2$, the proof follows from the above theorem.

Theorem 2.13. If $G$ is a connected graph, then

$$
\gamma_{\text {cnve }}(G) \leq \gamma^{\prime}(G)+2 \Delta(G)-1 \text {. }
$$

Proof: Let $E^{\prime}=\left\{e_{1}=x_{1} y_{1}, e_{2}=x_{2} y_{2}, \ldots, e_{\gamma^{\prime}(G)}=x_{\gamma^{\prime}(G)} y_{\gamma^{\prime}(G)}\right\}$ be a minimum edge dominating set for $G$. Let $e_{i}\left(1 \leq i \leq \gamma^{\prime}(G)\right)$ be any edge in $E^{\prime}$. Then $\left\{x_{1}, x_{2}, x_{3} \ldots, x_{\gamma^{\prime}(G)}, N\left(e_{i}\right)\right\}$ is a cnved-set for $G$ whose cardinality is $\gamma^{\prime}(G)+d\left(e^{\prime}\right)-1$. Hence

$$
\begin{aligned}
& \gamma_{\text {cnve }}(G) \leq \gamma^{\prime}(G)+d\left(x_{i}\right)+d\left(y_{i}\right)-1 \\
& \leq \gamma^{\prime}(G)+2 \Delta(G)-1
\end{aligned}
$$

Note: The bound is sharp as it is attained in the case of $K_{2}$.

Theorem 2.14. If $G$ is a tree with $n$ vertices and $\operatorname{diam}(G) \geq 3$, then $\gamma_{\text {cnve }}(G)<n-\nu^{\prime}+\Delta(G)$, where $\nu^{\prime}$ is the number of pendant vertices in $G$.

Proof: Clearly the non pendant vertices of $G$ along with all the neighbours of a support vertex $v($ say) forms a cnved - set for $G$, whose cardinality is $n-\nu^{\prime}+d(v)$. Hence the inequality follows.

Theorem 2.15. If $G$ is a connected graph such that $\bar{G}$ (the complement of $G)$ is connected, then $\gamma_{\text {cnve }}(G)+\gamma_{\text {cnve }}(\bar{G}) \leq \frac{5 n}{2}+2(\Delta(G)-\delta(G))$.

Proof: By the Remark in Theorem.2.10,

$$
\begin{aligned}
& \gamma_{\text {cnve }}(G) \leq \gamma(G)+2 \Delta(G) \\
& \gamma_{\text {cnve }}(\bar{G}) \leq \gamma(\bar{G})+2 \Delta(\bar{G})
\end{aligned}
$$


So,

$$
\begin{aligned}
\gamma_{\text {cnve }}(G)+\gamma_{\text {cnve }}(\bar{G}) & \leq \gamma(G)+\gamma(\bar{G})+2(\Delta(G)+\Delta(\bar{G})) \\
& \leq \frac{n}{2}+2+2(\Delta(G)+n-\delta(G)-1)(\text { see }[3]) \\
& \leq \frac{5 n}{2}+2(\Delta(G)-\delta(G))
\end{aligned}
$$

Theorem 2.16. If $G$ is a connected graph with $\gamma(G)>3$, then $\gamma_{\text {cnve }}(G)+\gamma_{\text {cnve }}(\bar{G}) \leq 3(n-\delta(G))+2 \Delta(G)$.

Proof: By the Remark in Theorem.2.10 and [2] the proof follows.

Theorem 2.17. If $G$ is a connected graph with $\gamma(\bar{G})>3$, then

$$
\gamma_{\text {cnve }}(G)+\gamma_{\text {cnve }}(\bar{G}) \leq 2 \Delta(G)-\delta(G)+3 .
$$

Proof: By the Remark in Theorem.2.10 and [2] the proof follows.

Theorem 2.18. If $G$ is a connected graph such that $\bar{G}$ is connected, then $\gamma_{\text {cnve }}(G)+\gamma_{\text {cnve }}(\bar{G}) \leq(n-1)(n-2)+2$.

Proof: For any graph $G$,

$$
\begin{aligned}
\gamma_{\text {cnve }}(G) & \leq n \\
& =2(n-1)-n+2
\end{aligned}
$$

$\leq 2 \epsilon-n+2$ So,

Similarly, $\gamma_{\text {cnve }}(\bar{G}) \leq 2 \epsilon^{\prime}-n+2\left(\right.$ here $\epsilon^{\prime}$ is the number of edges in $\left.\bar{G}\right)$.

$$
\begin{aligned}
& \quad \gamma_{\text {cnve }}(G)+\gamma_{\text {cnve }}(\bar{G}) \leq 2\left(\epsilon+\epsilon^{\prime}\right)-2(n-2) \\
& \leq n(n-1)-2(n-2) \\
& =(\mathrm{n}-1)(\mathrm{n}-2)+2
\end{aligned}
$$

Note: If $G$ is a connected graph with $n$ vertices, then $\gamma_{\text {cnved }}(G) \leq n$. 
Now, we characterize the graphs for which $\gamma_{c n v e}(G)=n$.

Theorem 2.19. For a connected graph $G$ with $n$ vertices, $\gamma_{\text {cnved }}(G)=n$ iff for each edge $v_{1} v_{2}$ in $G, N\left(v_{1}\right) \cup N\left(v_{2}\right)=V$.

Proof: Assume that $\gamma_{\text {cnve }}(G)=n$. Suppose that there is an edge $v_{1} v_{2}$ in $G$ such that $N\left(v_{1}\right) \cup N\left(v_{2}\right) \neq V$. Consider the set $V-\left[N\left(v_{1}\right) \cup N\left(v_{2}\right)\right]$. Let $E^{\prime}=\left\{u v: u, v \in V-\left[N\left(v_{1}\right) \cup N\left(v_{2}\right)\right]\right\}$.

If $E^{\prime}=\phi$, then $\left[N\left(v_{1}\right) \cup N\left(v_{2}\right)\right](\subset V)$ is a cnved-set of $G$ whose cardinality is less than $n$.

Suppose $E^{\prime} \neq \phi$.

Let $E^{\prime}=\left\{x_{1} y_{1}, x_{2} y_{2}, \ldots, x_{s} y_{s}\right\}$. Then $\left[N\left(v_{1}\right) \cup N\left(v_{2}\right)\right] \bigcup\left\{x_{1}, x_{2}, \ldots, x_{s}\right\}$ is a cnved-set of $G$ whose cardinality is less than $n$.

Hence in either case we get a contradiction to our assumption.

Assume that the converse holds. Let $D$ be the minimum cnved-set of $G$. By the Characterization Result for cnved-set there is an edge $v_{1} v_{2}$ such that the neighbours of $v_{1}, v_{2}$ are from $D$. Then by our assumption $N\left(v_{1}\right) \cup N\left(v_{2}\right)=V$. Hence $\gamma_{c n v e}(G)=n$.

Corollary 2.20. If $G=C_{n}$, then $\gamma_{\text {cnve }}(G)=n$ iff $n=3,4$.

Corollary 2.21. If $G=P_{n}$, then $\gamma_{\text {cnve }}(G)=n$ iff $n=2,3$.

\section{Corollary 2.22.}

1. $\gamma_{\text {cnve }}\left(S_{n}\right)=n, n \geq 3$

2. $\gamma_{\text {cnve }}\left(K_{n}\right)=n, n \geq 3$

3. $\gamma_{\text {cnve }}\left(K_{m, n}\right)=m+n$

Theorem 2.23. Let $G$ be a connected graph with $\operatorname{diam}(G)=2$ and circumference of $G$ is three, then $\gamma_{c n v e}(G) \neq n$ iff there is a triangle $T$ in $G$ for which there is a vertex in $V-V(T)$ adjacent to exactly one vertex in $T$.

Proof: Assume that $\gamma_{\text {cnve }}(G) \neq n$. 
By Theorem.2.19. there is an edge $u v$ in $G$ such that $N[u] \cup N[v] \neq V$. Since $\operatorname{diam}(G)=2$ and $c(G)=3$, for $x \in V-[N[u] \cup N[v]]$ there is $w \in N[u] \cap N[v]$ such that $w x$ is an edge in $G$. So $\langle u v w>(=T)$ is a triangle in $G$. Supposing that all the vertices in $V-V(T)$ are adjacent to atleast two vertices in $T$ we get a contradiction to that $N[u] \cup N[v] \neq V$. Hence there is atleast one vertex in $V-V(T)$ which is adjacent to exactly one vertex in $T$.

The converse part is clear.

Theorem 2.24. If $G$ is a connected graph such that $\bar{G}$ is connected and $\operatorname{diam}(G) \geq 4$, then $\gamma_{\text {cnve }}(G)+\gamma_{\text {cnve }}(\bar{G}) \leq(n-1)(n-2)$.

Proof: By Theorem.2.19, observe that for any graph $G$,

$$
\gamma_{\text {cnve }}(G) \leq n-1
$$

Now we construct the proof as in the case of Theorem.2.18.

Corollary 2.25. $G$ be a tree with $n$ vertices, then $\gamma_{c n v e}(G)=n$ iff $G \cong S_{n}$.

Proof: Assume that $\gamma_{\text {cnve }}(G)=n$. Then by the Characterization Result for each edge $v_{1} v_{2}$ in $G, N\left(v_{1}\right) \cup N\left(v_{2}\right)=V$. So for any pendant edge $u v$ in $G, N(u) \cup N(v)=V$, which implies one of $u, v$ is adjacent to all the vertices in $G$. W.l.g assume that $v$ is the vertex adjacent to all the vertices in $G$ (i.e $u$ is a pendant vertex). Since $G$ is a tree there is no edge between $v_{1}, v_{2} \in V-\{v\}$ (i.e. all the vertices in $V-\{v\}$ are pendant). Hence $G \cong S_{n}$.

For the converse part, any edge $u v$ in $S_{n}$ has the property that $N(u) \cup N(v)=$ $V$. Hence by the Characterization Result the claimant holds.

Now we characterize the graphs for which $\gamma_{\text {cnve }}(G)=3$.

Theorem 2.26. $G$ be a tree, then $\gamma_{c n v e}(G)=3$ iff $G=P_{3}$ or $G$ is obtained by adding zero or more leaves to exactly one pendant vertex of $P_{4}$.

Proof: Assume that $\gamma_{c n v e}(G)=3$. Let $D=\left\{v_{1}, v_{2}, v_{3}\right\}$ be a minimum cnved-set of $G$. Then by the Characterization Result $\langle D\rangle$ is connected .

Clearly atleast one of $v_{1}$ or $v_{3}$ is a pendant vertex.

Suppose both $v_{1}, v_{3}$ are pendant vertices. 
If there is any vertex in $G$ adjacent to $v_{2}$ then it should be a member of $D$, which is a contradiction to our assumption. Hence $G=P_{3}$.

Suppose exactly one of $v_{1}, v_{3}$ is a pendant vertex.

W.l.g assume that $v_{1}$ is the pendant vertex. Clearly no vertex other than $v_{1}, v_{3}$ can be adjacent with $v_{2}$. Hence any vertex or edge in $<V-$ $\left\{v_{1}, v_{2}, v_{3}\right\}>$ is adjacent to $v_{3}$. Then $G=P_{4}$ or $G$ is obtained by adding zero or more leaves to exactly one pendant vertex of $P_{4}$.

The converse part is clear.

Theorem 2.27. $G$ be a connected graph with $\delta(G) \geq 2$. Then $\gamma_{c n v e}(G)=$ 3 iff

(i) there is a $C_{3}$ edge disjoint with the other cycles in $G$.

(ii) each edge in $G$ lies on a cycle of length atmost four that has a common vertex with $C_{3}$.

Proof: Assume that $\gamma_{\text {cnve }}(G)=3$.

Let $D$ be a minimum cnved- set of $G$. By the Characterization Result and by the hypothesis,

$<D>=C_{3}=\langle u v w\rangle$ (say). By our assumption none of the edges of $C_{3}$ are common to two cycles. Hence $C_{3}$ is edge disjoint with the remaining cycles in $G$. If any edge in $G$ lies on a cycle of length greater than four then there is atleast one edge not dominated by the vertices of $D$. Hence $(i i)$ holds.

Conversely let $C_{3}$ be the cycle through $u, v, w$ satisfying conditions (i)\&(ii).

Denote $D=\{u, v, w\}$. Let $v$ be the vertex in $C_{3}$ through which the vertices in $V-V\left(C_{3}\right)$ reach the vertices $\{u, w\}$. Let $v_{1} v_{2}$ be an any edge in $G$. Then by $(i i)$ of our assumption, $v_{1} v_{2}$ lies on a cycle of length three or four for which $v$ is one of the vertices. In either case $v_{1} v_{2}$ is ve dominated by $v$. Hence $D$ is a cnved-set. Since $\delta(G) \geq 2, D$ is the $\gamma_{c n v e}(G)-$ set of cardinality 3 .

This proves the result.

$G$ is a union of edge disjoint triangles, where all the triangles having a common vertex, then $\gamma_{\text {cnve }}(G)=3$. 
Theorem 2.29. $G$ be a tree and $D$ be the set of all pendant vertices in $G$. Then $D$ is a cnved-set iff $G=P_{2}$.

Proof: Suppose that $D$ is cnved-set of $G$. Then by the Characterization Result for cnved-set, there is an edge $v_{1} v_{2}\left(v_{1}, v_{2} \in D\right)$ in $G$ all of whose neighbours are from $D$. This implies $v_{1}, v_{2}$ are pendant vertices. Hence $G=P_{2}$.

The converse part is clear.

Theorem 2.30. $G$ be a tree with $n$ vertices, then $\gamma_{c n v e}(G)=n-2$ iff $G=P_{5}$ or $G=P_{6}$ or $G=S_{1,2}$ or $G=S_{2,2}$.

Proof: Assume that $\gamma_{\text {cnve }}(G)=n-2$.

Suppose $\operatorname{diam}(G)=k$ where $k \geq 6$.

If any two pendant vertices $v_{1}, v_{2}$ in $G$ are adjacent(with $v_{3}$ ), then $V-\left\{v_{1}, v_{2}, v_{3}\right\}$ is a cnved-set of cardinality $n-3$ which is a contradiction to our assumption.

Suppose that $P=\left\langle v_{1} v_{2} v_{3} \ldots . v_{k-1} v_{k}\right\rangle$ be the diammetral path in $G$. Then $V-\left\{v_{1}, v_{2}, v_{k-1}, v_{k}\right\}$ is a cnved-set of $G$ of cardinality less than $n-2$ leading to a contradiction.

Hence $\operatorname{diam}(G)<6$.

Suppose $\operatorname{diam}(G)=5$.

If $G$ has exactly two pendant vertices, then $G=P_{6}$, which implies $\gamma_{\text {cnve }}(G)=n-2$. Suppose $G$ has more than two pendant vertices. Clearly no two pendant vertices in $G$ have a common neighbour.

Let $P=\left\langle v_{1} v_{2} v_{3} v_{4} v_{5} v_{6}\right\rangle$ be the diammetral path in $G$. Any vertex in $V-\left\{v_{1}, v_{2}, v_{3}, v_{4}, v_{5}, v_{6}\right\}$ can be adjacent to $v_{3}$ or $v_{4}$. If $v_{7}$ is a pendant vertex not adjacent to $v_{3}$ or $v_{4}$ then there is a path from $v_{1}$ to $v_{7}$ through $v_{3}$ or a path from $v_{6}$ to $v_{7}$ through $v_{4}$. In any case $V-\left\{v_{1}, v_{2}, v_{5}, v_{6}\right\}$ is a cnved-set of cardinality less than $n-2$ which contradicts our assumption. If each vertex in $V-\left\{v_{1}, v_{2}, v_{3}, v_{4}, v_{5}, v_{6}\right\}$ is adjacent to $v_{3}$ or $v_{4}$, then $V-\left\{v_{1}, v_{5}, v_{6}\right\}$ is a cnved-set of cardinality less than $n-2$ which is a contradiction.

Hence when $\operatorname{diam}(G)=5, G=P_{6}$. 
Suppose $\operatorname{diam}(G)=4$.

If $G=P_{5}$, then $\gamma_{\text {cnve }}(G)=n-2$. Suppose $G \neq P_{5}$. Clearly no two pendant vertices are adjacent. Let $P=\left\langle v_{1} v_{2} v_{3} v_{4} v_{5}>\right.$ be the diammetral path in $G$. Then any vertex in $V-\left\{v_{1}, v_{2}, v_{3}, v_{4}, v_{5}\right\}$ is adjacent to $v_{3}$ or a pendant vertex at a distance two from $v_{3}$. In the later case $V-$ $\left\{v_{1}, v_{2}, v_{4}, v_{5}\right\}$ is a cnved-set of cardinality $n-4$ which contradicts our assumption. In the former case $V-\left\{v_{1}, v_{2}, v_{3}\right\}$ is cnved-set of cardinality $n-3$ which again contradicts our assumption.

Hence when $\operatorname{diam}(G)=4, G=P_{5}$.

Suppose $\operatorname{diam}(G)=3$.

If $G=P_{4}$, then $\gamma_{\text {cnve }}(G)=n-1>n-2$, which is a contradiction.

Suppose $G \neq P_{4}$. Then $G \cong S_{p, q}(p+q \geq 3)$. If $\max \{p, q\} \geq 3$, then $\gamma_{\text {cnve }}(G)=n-\max \{p, q\}<n-2$, which is a contradiction. Therefore $\max \{p, q\}=2$. Then $G=S_{1,2}$ or $G=S_{2,2}$. In any case $\gamma_{\text {cnve }}(G)=n-2$.

Hence when $\operatorname{diam}(G)=4, G=S_{1,2}$ or $G=S_{2,2}$.

If $\operatorname{diam}(G) \leq 2$, then by Theorem.2.19. $\gamma_{\text {cnve }}(G)=n$.

The converse part is clear.

Theorem 2.31. $G$ be a tree with $n$ vertices, then $\gamma_{\text {cnve }}(G)=n-1$ iff $G=P_{4}$.

Proof: Suppose that $\gamma_{\text {cnve }}(G)=n-1$.

Suppose $\operatorname{diam}(G) \geq 6$.

Let $P=<v_{1} v_{2} v_{3} v_{4} \ldots v_{k}>(k \geq 7)$ be a diammetral path in $G$. Then $V-\left\{v_{1}, v_{k}\right\}$ is a cnved-set of $G$

$$
\Rightarrow \gamma_{\text {cnve }}(G) \leq n-2<n-1, \text { a contradiction. }
$$

Hence $\operatorname{diam}(G)<6$.

Suppose $\operatorname{diam}(G)=5$.

If $G=P_{6}$, then $\gamma_{\text {cnve }}(G)=n-2$. So $G \neq P_{6}$. Clearly any two pendant vertices in $G$ do not have a common neighbour. Let $v$ be an arbitrary vertex in $V-\left\{v_{1}, v_{2}, v_{3}, v_{4}, v_{5}, v_{6}\right\}$. Then

(i) $v$ can be adjacent to $v_{3}$ or $v_{4}$

(ii) $v$ is at a distance three from $v_{3}$ or $v_{4}$. 
In any of the cases, $\gamma_{\text {cnve }}(G)=n-2<n-1$.

Suppose $\operatorname{diam}(G)=4$.

If $G=P_{5}$, then $\gamma_{c n v e}(G)=n-2$. So $G \neq P_{5}$. Clearly any two pendant vertices in $G$ do not have a common neighbour. Let $v$ be an arbitrary vertex in $V-\left\{v_{1}, v_{2}, v_{3}, v_{4}, v_{5}\right\}$. Then $v$ is adjacent to $v_{3}$ or at a distance two from $v_{3}$. In any case $\gamma_{\text {cnve }}(G)=n-2<n-1$.

Suppose $\operatorname{diam}(G)=3$.

If $G=P_{4}$, then $\gamma_{c n v e}(G)=n-1$. Suppose $G \neq P_{4}$. This implies there is a pair of pendant vertices in $G$ having a common neighbour. Then $\gamma_{\text {cnve }}(G)<n-1$.

If $\operatorname{diam}(G) \leq 2$, then $\gamma_{\text {cnve }}(G)=n>n-1$.

The converse part is clear.

\section{Proposition 2.31.}

1. $\gamma_{c n v e}\left(S_{n, m}\right)=\min \{n+1, m+1\}$

2. $\gamma_{\text {cnve }}\left(C_{n}\right)=n, n \geq 3$

3. $\gamma_{\text {cnve }}\left(P_{n}\right)=n$

Result 2.33. $G$ be connected graph and $D$ be a cnved-set of $G$. If $E^{\prime}=$ $\{u v / u \in D$ or $v \in D\}$, then the minimum edge domination number is atmost $\left|E^{\prime}\right|-1$.

Proof: Assume that the hypothesis holds. Clearly $E^{\prime}$ is an edge dominating set of $G$. Since $D$ is a cnved-set of $G$ there is $v_{1} v_{2}$ in $E(G)$ such that $N\left[v_{1}\right] \subseteq D ; N\left[v_{2}\right] \subseteq D \Rightarrow v_{1} v_{2} \in E^{\prime}$. Clearly $E^{\prime}-\left\{v_{1} v_{2}\right\}$ is also an edge dominating set of $G$. Hence min edge domination number is atleast $\left|E^{\prime}\right|-1$.

Result 2.34. $G$ be a connected graph and $D$ be a cnved-set. Then $D$ is connected ved-set of $G$ iff $\langle D>$ has exactly one component and $|D| \geq 3$.

Proof: Suppose that the hypothesis holds. Assume that $D$ is connected ved-set of $G$. Then $\langle D\rangle$ has exactly one component. By the nature of $D$, it has atleast three vertices. Converse is clear. 


\section{References}

[1] Bondy J. A. and Murthy, U. S. R., Graph theory with Applications, The Macmillan Press Ltd, (1976).

[2] Danut Marcu, A Note On the Domination Number Of a Graph and its Complement. MATHEMATICA BOHEMICA, Vol. 126 (1), pp. 63-65, (2001).

[3] T. W.Haynes, S. T. Hedetneimi, P.J. Slater, Fundamentals of Dominations in Graphs, Marcel Dekker, New York, (1988).

[4] J. D.Horton, K. Kilakos, Minimum edge dominating sets. SIAM J.Discrete Math., Vol. 6 (3), pp. 375-387, (1993).

[5] R. Laskar, K. Peters, Vertex and edge dominating parameters in Graphs. Congr.Numer., Vol. 48, pp. 291-305, (1985).

[6] T. Tamizh Chelvam, S. Robinson Chellathurai,Complementary Nil Domination Number of a Graph. Tamkang Journal Of Mathematics., Vol. 40 (2), pp. 165-172, (2009).

\section{S. V. Siva Rama Raju}

Department of Mathematics

Ibra College of Technology

Ibra, Sultanate of Oman

e-mail:shivram2006@yahoo.co.in

and

\section{H. Nagaraja Rao}

Department of Mathematics

G. V. P. P. G. Courses

Visakhapatnam,India

e-mail:ihnrao@yahoo.com 